\title{
Diagnosis and Management of Monoclonal Gammopathy and Smoldering Multiple Myeloma
}

Timothy M. Schmidt, MD, ${ }^{1}$ and Natalie S. Callander, MD ${ }^{2}$

\section{ABSTRACT}

The presence of monoclonal proteins is common, with a prevalence in the United States around 5\% that increases with age. Although most patients are asymptomatic, most cases are caused by a clonal plasma cell disorder. Monoclonal gammopathy of undetermined significance (MGUS) and smoldering multiple myeloma (SMM) are asymptomatic precursor conditions with variable risk of progression to multiple myeloma. In recent years, significant progress has been made to better understand the factors that lead to the development of symptoms and progression to myeloma. This review summarizes the current diagnosis treatment guidelines for MGUS and SMM and highlights recent advances that underscore a shifting paradigm in the evaluation and management of plasma cell precursor conditions.

J Natl Compr Canc Netw 2020;18(12):1720-1729 doi: $10.6004 /$ jnccn. 2020.7660
${ }^{1}$ Winship Cancer Institute, Emory University, Atlanta, Georgia; and

${ }^{2}$ University of Wisconsin Carbone Cancer Center, Madison, Wisconsin.

\section{Monoclonal Gammopathy of Undetermined Significance}

\section{Background}

Paul Ehrlich is credited with developing the concept of immune cells producing anti-infective weaponry, which he termed "side chains," postulated to be receptors that could somehow be harnessed to fight infection. ${ }^{1}$ Since that important hypothesis was made in Germany in 1897, several other key developments occurred in the ensuing 50 years allowing the identification and measurement of such proteins and the cells of origin, now known as antibodies and plasma cells, respectively. With the advent of serum protein electrophoresis, pioneered by Tiselius ${ }^{2}$ and Longsworth et $\mathrm{al}^{3}{ }^{3}$ investigators quickly noted the occurrence of monoclonal antibodies in the sera of both ill and healthy people, a phenomenon termed "essential hyperglobulinemia" ${ }^{4}$ and documented by others. ${ }^{5}$

However, researchers are indebted to the painstaking work of Robert Kyle, who coined the term monoclonal gammopathy of undetermined significance (MGUS) and established the framework that is still in use today. ${ }^{6}$ His seminal publication tracked the outcome of 241 patients found to have serum monoclonal proteins but no evidence of multiple myeloma (MM) and carefully documented their course with at least 5 years of follow-up. Kyle found that most patients would never develop a symptomatic disorder. This review addresses MGUS and the related entity smoldering multiple myeloma (SMM).

The International Myeloma Working Group (IMWG) defines MGUS as the presence of a monoclonal protein (M protein), detected by either serum or urine protein electrophoresis, or unexplained free light chain (FLC) excess in the absence of a monoclonal IgH heavy chain, with serum monoclonal protein levels $<3 \mathrm{~g} / \mathrm{dL}$ and $<10 \%$ clonal plasma cells on bone marrow biopsy. ${ }^{7}$ In addition, the definition of MGUS requires that the individual have no symptoms of clinical myeloma (hypercalcemia, renal

See JNCCN.org for supplemental online content. 
dysfunction, anemia, and/or lytic bone disease [CRAB criteria]). MGUS incidence varies greatly based on ethnicity and age, with lower levels encountered in Asia, approximately $0.4 \%$ to $2 \%,{ }^{8,9}$ compared with those in Northern Europe and North America, with one study reporting an MGUS incidence of $6.1 \%$ in the United States. ${ }^{10}$ MGUS incidence increases with age ${ }^{11}$; it is found in $3 \%$ to $4 \%$ of the population aged 50 to 60 years compared with approximately $10 \%$ among octogenarians. Landgren et al ${ }^{12}$ found that in one portion of the NHANES study, which examined individuals aged 10 to 50 years, patients developed MGUS at a higher level but also at an earlier age compared with White and Mexican American individuals. Obesity has also been associated with an increased risk of MGUS ${ }^{13}$ and progression to MM. ${ }^{14}$

The search for a genetic cause of MGUS led to the discovery of hyperphosphorylated paratarg-7, a protein that may serve as an autoantigen driving chronic antigen stimulation and has been found in both familial and sporadic MGUS. The inheritance of paratarg-7 has been associated with a nearly 8-fold increased risk of developing MGUS $^{15}$ and seems to be detected more frequently in African Americans than in Whites with MGUS. ${ }^{16}$ Other loci that may be important in the development of MGUS and MM have been found in genome-wide association studies, including 3p22.1 (rs1052501), 6p21.33 (rs2285803), 7p15.3 (rs4487645), and 17p11.2 (rs4273077). ${ }^{17}$ Certain environmental exposures have also been associated with the development of MGUS, including Agent Orange, ${ }^{18}$ pesticides, ${ }^{19}$ and most recently, participation in the $9 / 11$ rescue operation. ${ }^{20}$

Because most individuals found to have MGUS will never go on to develop a symptomatic plasma cell disorder, routine screening for MGUS is not recommended. However, previous studies have found an increased risk of MGUS in first-degree relatives of patients with MM or other blood disorders. ${ }^{21-24}$ This risk seems even greater in first-degree relatives of African American patients with MM. Two large studies are underway to determine whether screening of these individuals will ultimately reduce the MM burden through early detection of precursor states: the Iceland Screens, Treats or Prevents Multiple Myeloma study (https://www.blodskimun.is) and the PROMISE study (https:/ /www.promisestudy.org). This effort is important because it is clear that MGUS nearly always precedes the development of MM..$^{25,26}$

\section{Diagnosis and Evaluation}

Diagnosis of MGUS requires the presence of a monoclonal protein, which is typically determined through serum protein electrophoresis or less often by urine protein electrophoresis and classified by immunofixation. MGUS is generally divided into 3 types: non-IgM MGUS (ie, IgG or IgA, or rarely IgD and IgE), IgM MGUS, and light-chain MGUS. Light-chain MGUS is defined as having an

\begin{tabular}{|c|c|}
\hline Diagnosis & Criteria (All Must be Met, Unless Otherwise Noted) \\
\hline Non-lgM MGUS & $\begin{array}{l}\text { 1. }<10 \% \text { BMPC } \\
\text { 2. Serum monoclonal protein (non-lgM) }<3 \mathrm{~g} / \mathrm{dL} \\
\text { 3. No SLiM-CRAB criteriaa }\end{array}$ \\
\hline IgM MGUS & $\begin{array}{l}\text { 1. }<10 \% \text { BMPC } \\
\text { 2. Serum IgM monoclonal protein }<3 \mathrm{~g} / \mathrm{dL} \\
\text { 3. No symptoms attributable to underlying } \\
\text { lymphoplasmacytic disorder (anemia, } \\
\text { hyperviscosity, adenopathy, organomegaly, or } \\
\text { constitutional symptoms) }\end{array}$ \\
\hline Light chain MGUS & $\begin{array}{l}\text { 1. Abnormal FLC ratio }(<0.26 \text { or }>1.65) \\
\text { 2. Increased level of the appropriate involved light } \\
\text { chain (increased } \kappa \mathrm{FLC} \text { in patients with ratio }>1.65 \\
\text { and increased } \lambda \mathrm{FLC} \text { in patients with ratio }<0.26) \\
\text { 3. No immunoglobulin heavy chain expression on } \\
\text { immunofixation } \\
\text { 4. Urinary monoclonal protein }<500 \mathrm{mg} / 24 \mathrm{~h} \\
\text { 5. }<10 \% \text { BMPC } \\
\text { 6. No SLiM-CRAB criteriaa }\end{array}$ \\
\hline MGRS & $\begin{array}{l}\text { 1. Presence of monoclonal immunoglobulin or light } \\
\text { chain } \\
\text { 2. Kidney biopsy showing renal impairment related } \\
\text { to the monoclonal protein }{ }^{28,97} \\
\text { 3. Diagnostic criteria for MM or other } \\
\text { lymphoplasmacytic disorder not met }\end{array}$ \\
\hline MGCS b & $\begin{array}{l}\text { 1. Presence of monoclonal immunoglobulin or light } \\
\text { chain } \\
\text { 2. Symptoms or clinical manifestations attributable } \\
\text { to the presence of monoclonal protein }{ }^{31} \\
\text { 3. Diagnostic criteria for MM or other } \\
\text { lymphoplasmacytic disorder not met }\end{array}$ \\
\hline SMM & $\begin{array}{l}\text { No SLiM-CRAB criteria }{ }^{a} \text { plus at least } 1 \text { of the } \\
\text { following: } \\
\text { 1. BMPC } \geq 10 \% \\
\text { 2. Serum } M \text {-spike } \geq 3 \mathrm{~g} / \mathrm{dL} \text { (or urine paraprotein } \\
\geq 500 \mathrm{mg} / 24 \mathrm{~h} \text { in the absence of a serum M-spike) }\end{array}$ \\
\hline MM & $\begin{array}{l}\text { 1. } B M P C \geq 10 \%^{c} \\
\text { 2. Presence of any SLiM-CRAB criteriaa }\end{array}$ \\
\hline
\end{tabular}

Abbreviations: BMPC, bone marrow plasma cells; FLC, free light chain; MGCS monoclonal gammopathy of clinical significance; MGRS, monoclonal gammopathy of renal significance; MGUS, monoclonal gammopathy of undetermined significance; MM, multiple myeloma; SMM, smoldering multiple myeloma.

aSerum calcium $>1 \mathrm{mg} / \mathrm{dL}$ higher than the upper limit of normal or $>11 \mathrm{mg} / \mathrm{d}$ ), creatinine clearance $<40 \mathrm{~mL} / \mathrm{min}$ or serum creatinine $>2 \mathrm{mg} / \mathrm{dL}$; hemoglobin $>2 \mathrm{~g} / \mathrm{dL}$ below the lower limit of normal, or a hemoglobin value $<10 \mathrm{~g} / \mathrm{dL}$; $\geq 1$ osteolytic lesion on skeletal radiography, CT, or PET/CT; BMPC $\geq 60 \%$; $\mathrm{FLC}$ ratio (involved/uninvolved) $\geq 100 ;>1$ focal lesion on MRI. bProposed.

If a patient has clear evidence of myeloma-defining events with $<10 \%$ BMPC, with no alternate explanation for these criteria, then it is reasonable to diagnose and treat as MM without meeting this criterion.

abnormal FLC ratio, elevation in the appropriate light chain, and the absence of an Ig heavy chain on immunofixation. ${ }^{27}$ Diagnostic criteria of these MGUS subtypes and other plasma cell precursor conditions are detailed in Table 1.

Recently, another entity associated with monoclonal protein has emerged: the monoclonal gammopathy of renal significance (MGRS), defined as a clonal proliferation that leads to the production of a nephrotoxic monoclonal protein or fragment but does not otherwise meet the criteria of other recognized diagnoses. ${ }^{28} \mathrm{~Pa}-$ tients with MGRS tend to have more proteinuria than 
those with typical MGUS and, by definition, some degree of renal impairment. The prevalence of this disorder is uncertain but may affect from $1.5 \%$ to $6 \%$ of patients with MGUS. ${ }^{29}$ To meet the definition of MGRS, a renal biopsy must be performed and carefully analyzed by immunohistochemistry and electron microscopy. Subtypes of MGRS include renal lesions such as immunotactoid glomerulonephritis, proliferative glomerulonephritis with monoclonal immunoglobulin deposits, and monoclonal gammopathy-associated C3 glomerulopathy. ${ }^{30}$ It is now recognized that in addition to these various renal manifestations, monoclonal proteins can, albeit rarely, present with a broad spectrum of clinical manifestations. Fermand et $\mathrm{al}^{31}$ proposed yet another clinical entity, monoclonal gammopathy of clinical significance (MGCS), which comprises a broad spectrum of symptomatic clinical syndromes related to an underlying $\mathrm{M}$ protein, frequently manifesting with neuropathy, vasculitis, or dermatologic changes.

Once patients are found to have a monoclonal protein, they must be evaluated with attention to any symptoms or other clinical elements that would potentially change management strategy. It can be challenging for clinicians to determine whether symptoms, which are often nonspecific, are related to the monoclonal protein or to other comorbidities; therefore, a careful and focused history and physical examination are required, with attention given to symptoms such as fatigue, pain, dyspnea, peripheral neuropathy, easy bruising, peripheral edema, nail or skin changes, and macroglossia. The presence of such symptoms may indicate that the patient has underlying MM, but can also be associated with conditions such as amyloid light-chain amyloidosis, POEMS syndrome (polyneuropathy, organomegaly, endocrinopathy, monoclonal protein, skin changes), or MGCS. Patients with MGUS with unexplained osteopenia should also be carefully scrutinized to make sure that bone loss is not actually related to undiagnosed MM. In addition, patients with some forms of non-Hodgkin lymphoma (NHL) and chronic lymphocytic leukemia (CLL) can present with small monoclonal proteins, and therefore these disease entities should be considered in the differential diagnosis. On physical examination, it is important to look for features such as an enlarged tongue, periorbital bruising, lymphadenopathy, organomegaly, and right-sided heart failure. Other suggested diagnostic testing is found in Table 2. Asymptomatic patients with low-risk MGUS, defined as those with an IgG isotype, a serum M-spike $<1.5 \mathrm{~g} / \mathrm{dL}$, a urine M-spike $<200 \mathrm{mg} / 24$ hours, and a normal FLC ratio, may be able to forgo a bone marrow biopsy and be observed, because results of the biopsy are unlikely to upstage them to either SMM or MM. ${ }^{32}$ In patients who do undergo a diagnostic bone marrow biopsy, those with $>5 \%$ plasma cells have a greater risk of progression to $\mathrm{MM}^{33}$ than those with a lower number of clonal plasma cells. Cytogenetic and fluorescence in situ hybridization (FISH) studies performed on those plasma cells may reveal alterations in the IgH locus, such as $t(11 ; 14)$ and trisomies, but no specific mutation reliably predicts progression from MGUS to myeloma. ${ }^{34}$ High-risk mutations such as a p53 deletion, $\mathrm{t}(4 ; 14)$, and $\mathrm{t}(14 ; 16)$ are less commonly encountered $\mathrm{d}^{35-37}$ among patients with MGUS.

Customarily, skeletal surveys have been used to screen for bone lesions in patients with MGUS; however, plain radiographs consistently underperform compared with newer techniques such as low-dose whole-body CT or MRI ${ }^{38-40}$ In patients with MGUS and higher levels of $M$ protein, increased FLC, or bony symptoms or advanced osteoporosis, some form of advanced imaging is preferred to rule out MM. ${ }^{41}$ For individuals with IgM MGUS, some investigators have recommended omitting bone imaging and instead using CT to search for adenopathy and organomegaly. ${ }^{42}$ Several investigations have found an increased risk of osteoporotic fractures in patients with MGUS, ${ }^{43-45}$ suggesting the potential utility of periodic bone density testing in patients with MGUS, although it is currently not considered routine practice.

\section{Follow-Up}

Once MGUS is discovered, patients do warrant follow-up because of the risk of disease progression to $\mathrm{MM}$ and Waldenström macroglobulinemia, as well as other illnesses, including NHL and CLL. A recent retrospective study using SEER data found that patients with MGUS who did not receive at least one follow-up visit had significantly higher rates of kidney failure, hypercalcemia, and fracture. ${ }^{46}$ In a study involving how to effectively triage patients with MGUS, Rajkumar et $\mathrm{al}^{47}$ evaluated a number of clinical features and found that individuals with an abnormal FLC ratio, a non-IgG monoclonal protein, or an $\mathrm{M}$ protein level $>1.5 \mathrm{~g} / \mathrm{dl}$ were at highest risk of progression; if all 3 characteristics were represented, then the risk of MM progression was $58 \%$ after 20 years. For reasons that are unclear, IgM MGUS is associated with approximately twice the risk of annual progression (ie, $1 \%$ to $2 \%$ per year). ${ }^{48}$ Therefore, patients with higher-risk MGUS (ie, presence of a higher M spike, abnormal FLC ratio, or non-IgG MGUS) should be followed serially, with a follow-up visit within 6 months of diagnosis. Because the risk of progression from MGUS to malignancy is greatest within the first year of diagnosis ${ }^{49}$ the follow-up period can then be extended to annual or semiannual if there has been no change in either M protein or FLC levels or other parameters (eg, appearance of anemia or renal insufficiency). Patients with low-risk MGUS can be followed less frequently, such as semiannually, after an initial follow-up 
Table 2. Suggested Initial Evaluation for MGUS and SMM

\begin{tabular}{|c|c|c|c|c|c|c|c|}
\hline & Asymptomatic & $\begin{array}{l}\text { Unexplained } \\
\text { Osteoporosis } \\
\text { Present }\end{array}$ & $\begin{array}{l}\text { Unexplained } \\
\text { Peripheral } \\
\text { Neuropathy }\end{array}$ & $\begin{array}{l}\text { Unexplained } \\
\text { Renal } \\
\text { Insufficiency }\end{array}$ & $\begin{array}{l}\text { Unexplained } \\
\text { Right-Sided } \\
\text { Heart Failure }\end{array}$ & $\begin{array}{l}\operatorname{lgM} \\
\text { MGUS }\end{array}$ & SMM \\
\hline $\mathrm{CBC}$ with differential & $x$ & $x$ & $x$ & $x$ & $x$ & $x$ & $x$ \\
\hline Creatinine, calcium, albumin, LDH & $x$ & $x$ & $x$ & $x$ & $x$ & $x$ & $x$ \\
\hline SPEP & $x$ & $x$ & $x$ & $x$ & $x$ & $x$ & $x$ \\
\hline Serum immunofixation & $x$ & $\mathrm{x}$ & $\mathrm{x}$ & $\mathrm{x}$ & $x$ & $\mathrm{x}$ & $\mathrm{x}$ \\
\hline FLCs and ratio & $x$ & $x$ & $\mathrm{x}$ & $x$ & $\mathrm{x}$ & $x$ & $\mathrm{x}$ \\
\hline UPEP, 24-h urine protein, and immunofixation & $x$ & $x$ & $x$ & $x$ & $x$ & $x$ & $x$ \\
\hline Troponin, NT-proBNP & - & - & $x$ & $x$ & $x$ & - & - \\
\hline Anti-MAG antibodies & - & - & $x$ & - & - & $x$ & - \\
\hline Electromyography & - & - & $x^{a}$ & - & - & - & - \\
\hline $\begin{array}{l}\text { Congo Red staining of skin biopsy or affected } \\
\text { organe }\end{array}$ & - & - & $x$ & $x$ & $x$ & - & - \\
\hline Advanced skeletal imaging ${ }^{f}$ & $x^{c}$ & $x$ & $x$ & $x$ & $x$ & - & $x$ \\
\hline Conventional CT scans & - & - & - & - & - & $x$ & - \\
\hline EKG and echocardiogram & - & - & - & - & $X^{9}$ & - & - \\
\hline
\end{tabular}

Abbreviations: EKG, electrocardiogram; FISH, fluorescence in situ hybridization; FLC, free light chain; LDH, lactate dehydrogenase; MAG, myelin-associated glycoprotein; MGUS, monoclonal gammopathy of undetermined significance; NT-proBNP, N-terminal pro-brain natriuretic peptide; SMM, smoldering multiple myeloma; SPEP, serum protein electrophoresis; UPEP, urine protein electrophoresis; VEGF, vascular endothelial growth factor.

aTypically in consultation with neurology.

blf involved light chain is lambda, to evaluate for POEMS (polyneuropathy, organomegaly, endocrinopathy, monoclonal protein, skin changes).

cMay be omitted if all criteria are met: IgG isotype, serum M-spike $<1.5 \mathrm{~g} / \mathrm{dL}$, 24-hour urine paraprotein $<200 \mathrm{mg}$, and normal FLC ratio.

Includes del(13), del(17p), $t(4 ; 14), t(11 ; 14), t(14 ; 16), t(14 ; 20),+1 q$, and del(1p).

eln addition to dedicated Congo Red staining for symptomatic patients, Congo Red staining of the bone marrow to screen for amyloidosis is reasonable if a first-time bone marrow biopsy is performed for any patient with a monoclonal protein.

fWhole-body low-dose CT preferred. Whole-body MRI, MRI of spine and bony pelvis, and ${ }^{18} \mathrm{FDG}-\mathrm{PET} / \mathrm{CT}$ are acceptable alternatives (listed in preferred order) if whole-body low-dose CT is not available. Bone survey is no longer an acceptable imaging modality unless no alternative exists.

9Cardiac MRI may be a more sensitive test that can be ordered depending on initial findings and suspicion of cardiac amyloidosis. Technetium pyrophosphate scanning can be used to look for non-amyloid light-chain cardiac amyloidosis.

shows stability, and often by a primary care provider rather than a specialist. Referral back to a hematologist/oncologist is warranted in the event of any significant changes, such as the appearance of anemia, renal insufficiency, or a significant increase in $\mathrm{M}$ protein level. Long-term follow-up of patients with MGUS has shown that they experience greater mortality from diseases such as CLL and NHL, but also from other solid tumors and cardiovascular disease, compared with matched control patients. ${ }^{50}$ However, at this time, no additional health screenings are recommended.

To date, no data suggest that routine treatment of MGUS is beneficial, and it should not be attempted outside of a clinical trial. There are some exceptions to this rule. Patients with IgM MGUS-related peripheral neuropathy and anti-myelin-associated globulin antibodies may benefit from interventions that target the abnormal B-cell clone. ${ }^{51}$ Such treatment may prevent progression of peripheral neuropathy. In addition, patients with MGRS are increasingly thought to require therapy directed toward elimination of the clone, causing the specific nephrotoxic immunoglobulins to protect renal function.

\section{Smoldering Multiple Myeloma}

\section{Background}

SMM is a clonal plasma cell disorder of intermediate risk existing on a continuum of malignant potential between MGUS and MM. After its initial description in $1980,{ }^{52}$ the definition of SMM was subsequently defined by the IMWG as a clonal plasma cell disorder with $\geq 10 \%$ clonal bone marrow plasma cells (BMPCs) and/or a serum paraprotein level of $\geq 3 \mathrm{~g} / \mathrm{dL}$ (or a urine paraprotein level of $\geq 500 \mathrm{mg} / 24$ hours in the absence of a serum M-spike) with no CRAB criteria or pathologic features of amyloidosis. ${ }^{53}$ In 2014, the IMWG updated the diagnostic criteria for $\mathrm{MM}$ to include patients with asymptomatic disease meeting $\geq 1$ "SLiM" criteria (ie, $\geq 60 \%$ plasma cells, light chain ratio involved:uninvolved $\geq 100: 1$, and 
MRI with $>1$ focal lesion) as having myeloma-defining events and recommended treatment initiation for these patients because of an unacceptably high risk of progression to symptomatic disease. ${ }^{7}$ This recommendation was based on data suggesting that the 2-year risk of progression to symptomatic myeloma is approximately $70 \%$ for patients with $\geq 2$ focal skeletal lesions on MRI, ${ }^{54,55} 64 \%$ to $72 \%$ among those with a serum FLC ratio of $\geq 100$ or $\leq 0.01,{ }^{56,57}$ and $95 \%$ for those with $\geq 60 \%$ BMPCs. ${ }^{56,58}$ Current diagnostic criteria for MGUS, SMM, and MM are displayed in Table 1. For decades, SMM has been managed essentially as highrisk MGUS, but new insights into disease biology and the development of highly effective and well-tolerated treatments for MM have generated substantial interest in determining whether there may be patients with SMM who would benefit from early intervention. Because the risk of progression to MM can be highly variable, it is important to identify which patients with SMM are at highest risk of progression to MM so that end-organ damage can avoided.

\section{Risk of Progression}

Among all patients with SMM, the risk of progression to symptomatic myeloma is estimated at approximately $10 \%$ per year for the first 5 years after diagnosis. ${ }^{59}$ However, there is significant heterogeneity regarding the risk of progression for individual patients; several models have been proposed to characterize this risk. A Spanish myeloma group published the first prediction model, which incorporated 2 risk factors: (1) the percentage of BMPCs with aberrant immunophenotype by flow cytometry, and (2) immunoparesis, or a reduction in the quantity of uninvolved (ie, non-paraprotein) immunoglobulins. $^{60}$ In 2008, a group from the Mayo Clinic published a separate model using clinical features, specifically the percentage of BMPCs $(\geq 10 \%)$, a serum M-spike ( $\geq 3 \mathrm{~g} / \mathrm{dL}$ ), and an abnormal serum FLC ratio $(\leq 0.125$ or $\geq 8)$ as risk factors for progression. ${ }^{61}$ This model became widely accepted because of its easy application and reliability in discriminating outcomes. In 2018, the Mayo Clinic group updated its diagnostic criteria, now referred to as the " $20 / 2 / 20$ " criteria, which redefined the cutoff values as BMPCs $\geq 20 \%$, an M-spike of $\geq 2 \mathrm{~g} / \mathrm{dL}$, and an FLC ratio $\geq 20 .{ }^{62}$ An area-under-thecurve analysis determined that the new criteria had a superior ability to accurately prognosticate the risk of progression to MM compared with the 2008 criteria. The IMWG subsequently validated this $20 / 2 / 20$ model in a cohort of 2,004 patients, showing the ability of the model to discriminate prognosis among these 3 categories, with a 2 -year risk of progression of $5 \%$, $17 \%$, and $47 \%$ among low-, intermediate-, and high-risk patients, respectively. ${ }^{63}$ A summary of these models and their prognostic risk calculation is provided in Table 3.

One of the most striking features of these prognostic models is that even among patients in the low-risk subsets, the risk of progression to $\mathrm{MM}$ is the greatest within the first 2 years after diagnosis. However, among patients who have not progressed to MM by 5 years, the annual risk decreases substantially. This observation suggests that there are at least 2 different patterns of disease biology among diagnoses of SMM: one pattern represents "presymptomatic myeloma" that could benefit from early intervention to prevent end-organ damage, and the other represents "MGUS-like" disease that follows an indolent course.

Several other clinical and biological factors have been evaluated as prognostic markers for progression to MM. Multiple studies have shown that certain cytogenetic abnormalities, including $\mathrm{t}(4 ; 14)$, del $(17 \mathrm{p}),+1 \mathrm{q}$, and hyperdiploidy, confer a higher risk of progression to symptomatic MM. ${ }^{64,65}$ These adverse cytogenetic abnormalities were an independent risk factor for progression in the Mayo Clinic 2018 cohort, ${ }^{62}$ but the number of patients who were evaluable was too small for inclusion in the model. Alterations in gene expression, such as myc activation, ${ }^{66,67}$ and high-risk gene expression profiling by GEP- $70^{68}$ have also been associated

Table 3. Risk Models in Smoldering Myeloma

\begin{tabular}{|c|c|c|c|}
\hline Model & $\mathbf{n}$ & Risk Factors & Risk of Progression \\
\hline PETHEMA study (Pérez-Persona et al, $2007^{60}$ ) & 93 & $\begin{array}{l}\text { 1. }>95 \% \text { of BMPC with aberrant markers by } \\
\text { flow cytometry } \\
\text { 2. Immunoparesis, defined as reduction of } \geq 1 \\
\text { uninvolved heavy chains in the serum to }<\text { LLN }\end{array}$ & $\begin{array}{l}0 \text { factors: } 4 \% \text { at } 5 y \\
1 \text { factor: } 46 \% \text { at } 5 y \\
2 \text { factors: } 72 \% \text { at } 5 y\end{array}$ \\
\hline Mayo Clinic study (Dispenzieri et al, 2008 ${ }^{61}$ ) & 273 & $\begin{array}{l}\text { 1. } \mathrm{BMPC} \geq 10 \% \\
\text { 2. Serum } \mathrm{M} \text {-spike } \geq 3 \mathrm{~g} / \mathrm{dL} \\
\text { 3. FLC ratio } \leq 0.125 \text { or } \geq 8\end{array}$ & $\begin{array}{l}1 \text { factor: } 12 \% \text { at } 2 y, 25 \% \text { at } 5 y, 50 \% \text { at } 10 y \\
2 \text { factors: } 27 \% \text { at } 2 y, 51 \% \text { at } 5 y, 65 \% \text { at } 10 y \\
3 \text { factors: } 52 \% \text { at } 2 y, 76 \% \text { at } 5 y, 84 \% \text { at } 10 y\end{array}$ \\
\hline Mayo Clinic study (Lakshman et al, 201862) & 421 & $\begin{array}{l}\text { 1. } \text { BMPC } \geq 20 \% \\
\text { 2. Serum } M-s p i k e ~ \geq 2 \mathrm{~g} / \mathrm{dL} \\
\text { 3. FLC ratio } \leq 0.05 \text { or } \geq 20\end{array}$ & $\begin{array}{l}0 \text { factors: } 9.7 \% \text { at } 2 y, 22.5 \% \text { at } 5 y, 52.7 \% \text { at } 10 y \\
1 \text { factor: } 26.3 \% \text { at } 2 y, 46.7 \% \text { at } 5 y, 65.3 \% \text { at } 10 y \\
2-3 \text { factors: } 47.4 \% \text { at } 2 y, 81.5 \% \text { at } 5 y, 96.5 \% \text { at } 10 y\end{array}$ \\
\hline
\end{tabular}

Abbreviations: BMPC, bone marrow plasma cells; FLC, free light chain; LLN, lower limit of normal. 
with progression to MM. Evolving paraprotein is a dynamic marker that has been shown to indicate a very high risk of progression to symptomatic myeloma in multiple studies. ${ }^{69-71}$ Rajkumar et $\mathrm{al}^{72}$ proposed a set of criteria for high-risk SMM, defined as $\geq 10 \%$ BMPCs with any combination of proposed high-risk factors, including cytogenetics, gene expression profiling, and isotype. This definition has been used as broad inclusion criteria for clinical trial enrollment but has yet to be validated in an independent patient cohort and is not yet used broadly in clinical practice. It is likely that in the future, several of these factors will be incorporated into new risk models and management decisions for patients with SMM.

\section{Management of SMM}

The current standard of care for management of SMM, regardless of the risk of progression, is observation. However, this standard is now being challenged by exciting clinical trial data suggesting that early intervention can prevent, or at least delay, progression to symptomatic MM, particularly among patients at high risk. Two general strategies have been proposed and are being evaluated in multiple clinical trials: a prevention strategy using low-intensity therapy and a curative approach intended to eradicate the neoplastic clone with highintensity treatment.

The preventive approach is supported by substantial preclinical data suggesting profound immune dysregulation as the disease progresses from MGUS to SMM to MM. ${ }^{73-77}$ Lenalidomide is an immunomodulatory drug that exerts its antimyeloma activity through multiple mechanisms, including the enhancement of T-cell- and natural killer cell-mediated cytotoxicity against myeloma cells, ${ }^{78-81}$ and is a backbone of MM therapy. The QUIREDEX trial ${ }^{82}$ randomized patients to observation versus time-limited low-intensity therapy using lenalidomide and dexamethasone (Rd), followed by maintenance lenalidomide. In this study, Rd was well tolerated and resulted in improved progressionfree survival (PFS; median not reached vs 21 months; hazard ratio $[\mathrm{HR}], 0.18$ ) and 3-year overall survival (OS; $94 \%$ vs $80 \%$; HR, 0.31 ) compared with observation alone. However, this approach was not adopted into clinical practice, largely because the trial used an SMM definition not widely accepted in the United States and also did not require advanced imaging to rule out lytic lesions. As such, patients who were asymptomatic but actually had a myeloma-defining event were likely enrolled, accounting for the rapid progression to MM seen in the observation arm.

The phase III ECOG-E3A06 trial ${ }^{83}$ randomized patients with intermediate- or high-risk SMM based on Mayo Clinic 2008 criteria $^{61}$ to receive continuous lenalidomide monotherapy (25 $\mathrm{mg}$ on days $1-21$ of 28-day cycles) versus observation, and required advanced imaging during study enrollment to exclude patients with MM. In this study, despite a modest overall response rate of $50 \%$ with very few achieving a very good partial response or better, the lenalidomide group had a substantially superior PFS $(91 \%$ vs $66 \%$ at 3 years; HR, $0.28 ; P=.002$ ), with the most profound benefit seen among patients with high risk per the Mayo Clinic 2018 criteria (HR, 0.09). ${ }^{62}$ Grade 3/4 adverse events were observed in $41 \%$ of patients in the treatment arm, and although the trial was designed to use continuous therapy, more than half of the patients discontinued lenalidomide (median time on therapy was approximately 2 years), and $80 \%$ of the patients remaining on lenalidomide at 12 months required a dose reduction. The rate of secondary primary malignancies was $5.2 \%$ in the lenalidomide arm compared with $3.5 \%$ in the observation arm. Despite the added toxicity from lenalidomide, no change in healthrelated quality-of-life scores between the 2 arms was seen after 24 cycles. Although the data are too immature to show an impact on OS, this study overcame many of the limitations of the QUIREDEX study ${ }^{82}$ and provides a rationale for the use of lenalidomide as a treatment for high-risk SMM.

Daratumumab (Dara) is an anti-CD38 monoclonal antibody with multiple mechanisms ${ }^{84-87}$ that is well tolerated and widely used in the treatment of plasma cell disorders. The CENTAURUS study ${ }^{88}$ evaluated singleagent Dara in several dosing strategies for the treatment of SMM. Dara resulted in an overall response rate of $37.5 \%$ to $56.1 \%$ and a $24-$ month PFS of $75.6 \%$ to $87.8 \%{ }^{88}$ The AQUILA trial (ClinicalTrials.gov identifier: NCT03301220), which randomizes patients to subcutaneous Dara vs observation, and the phase III DETER-SMM trial (EAA173; NCT03937635), which randomizes patients to Dara/Rd versus Rd, are ongoing and will help further explore the optimal preventive approach to highrisk SMM. Additional phase II-III interventional studies are listed in supplemental eTable 1 (available with this article at JNCCN.org).

Contrary to the preventive strategy, a curative approach to SMM is backed by the rationale that long-term survival in MM is highly correlated with deep response to antimyeloma therapy, particularly measurable residual disease (minimal residual disease [MRD]) negativity. ${ }^{89-91}$ Proponents of this strategy have proposed that treatment using high-intensity therapy, such as triplet or quadruplet combination induction, with or without autologous stem cell transplantation (ASCT), may eradicate the precursor clone and result in a cure for a large subset of patients with SMM. In a phase II study of carfilzomib/lenalidomide/ dexamethasone (KRd) and 2 years of lenalidomide 
maintenance in patients with newly diagnosed high-risk $\mathrm{SMM},{ }^{92}$ all 18 patients achieved a very good partial response or better, and 10 of 18 continued to be MRDnegative by next-generation flow cytometry and had a 4-year PFS of $71 \%$ with a median follow-up of 43.3 months. The phase II GEM-CESAR study ${ }^{93}$ enrolled 90 patients with high-risk SMM to receive induction using KRd for 6 cycles, followed by ASCT, 2 cycles of KRd consolidation, and 2 years of maintenance lenalidomide. In that study, 30-month PFS was 93\%, and among patients who had finished consolidation, $\geq 70 \%$ achieved a complete response, with $57 \%$ achieving MRD negativity by next-generation flow cytometry. Eleven patients discontinued treatment, including 2 deaths, but the overall frequency of grade 3-4 adverse effects was low. The ASCENT trial (NCT03289299) is evaluating treatment using Dara/KRd for 12 cycles, followed by maintenance Dara/ lenalidomide. This multicenter phase II trial is still accruing and represents a nontransplant alternative with a curative approach.

Overall, recent data from both strategies (ie, limited low-intensity treatment vs aggressive, MM-like treatment) suggest that researchers and physicians are nearing a paradigm shift in the standard management of patients with SMM, particularly those who are at high risk of progression. Although there is some concern about treating a proportion of patients who may never experience progression to overt malignancy, it is likely that our ability to prognosticate progression will improve with the adoption of cytogenetics and/or molecular studies into risk stratification models. Furthermore, there are now multiple randomized phase III studies that show improved PFS with a lenalidomide-based preventive strategy, particularly among patients with high risk. Critics have expressed concern that lenalidomide has not clearly shown an OS benefit in SMM and that lowintensity therapy may select for a resistant and/or more aggressive clone in patients who experience progression to MM. Alternatively, a high-intensity approach may ultimately cure some patients, but with substantial toxicity compared with a prevention strategy. There are no randomized data to compare outcomes directly between these 2 treatment approaches. Response rates are much higher among the intensive-treatment regimens, with a high proportion achieving a deep response, including MRD negativity. However, because the frequency of progression events at 2 to 3 years is similar for both strategies, this tendency may indicate that in a precursor state, achievement of a deep response with MRD negativity may not be as important to long-term survival. ${ }^{94}$ Supporting this hypothesis are data showing that stemlike memory cells are enriched in the bone marrow microenvironment of patients with MGUS but absent in those with $M M{ }^{77}$ suggesting that immunomodulatory therapy may help achieve long-term disease control by reverting patients with "presymptomatic myeloma" to an "MGUS-like" state. Nonetheless, both strategies seem promising, and long-term follow-up of these highlighted trials will be required to determine if one strategy is superior to the other.

\section{Summary and Recommendations}

Before considering the optimal management strategy for treating SMM, the first priority must be to establish that the patient does in fact have SMM. In addition to measuring serum and urine paraprotein and evaluating for symptomatic myeloma, all patients with suspected SMM require a bone marrow biopsy, FLC measurement, and advanced imaging. We support the IMWG recommendation in favor of whole-body low-dose CT for all patients with suspected SMM, but acknowledge that this modality is unavailable in many areas; therefore, whole-body MRI, MRI of the spine and pelvis, and/or PET scan are reasonable alternatives. ${ }^{95}$ Patients who have a myeloma-defining event $(\geq 60 \%$ BMPCs, serum FLC ratio of $\geq 100$ or $\leq 0.01$, and/or $\geq 2$ focal bone lesions) should be considered as having symptomatic MM and should be treated as such. Cytogenetic evaluation of the bone marrow aspirate with a comprehensive myeloma FISH panel, including $\mathrm{t}(4 ; 14), \mathrm{t}(11 ; 14), \mathrm{t}(14 ; 16)$, $t(14 ; 20)$, gain(1q), del(17p), and hyperdiploidy, should be performed on every patient, because this information may provide important prognostic information and potentially impact future treatment decisions. A comprehensive summary of recommendations for the workup of patients with SMM can be found in the NCCN Clinical Practice Guidelines in Oncology for Multiple Myeloma. ${ }^{96}$

After confirming a diagnosis of SMM, the current standard of care is risk stratification and observation with monitoring for changes in the quantity of paraprotein or the development of CRAB criteria. We recommend surveillance every 3 to 6 months, with closer monitoring of patients who are at high risk, particularly with increasing parameters indicative of an evolving pattern. If a patient is estimated to have an approximately $50 \%$ risk of progression to MM in 2 years according to any of the models, then treatment with lenalidomide for 2 years can be considered, in light of results from the QUIREDEX ${ }^{82}$ and ECOG-E3A06 ${ }^{83}$ trials. However, note that although PFS data are encouraging, no OS data are currently available from the ECOG dataset. Furthermore, although the toxicity seen in that dataset was consistent with the known profile of lenalidomide, the high rate of attrition in the ECOG study shows that patients may be less willing to accept adverse effects when it is unknown whether an individual will ever progress to MM. Although a curative approach has theoretical appeal, until there are mature 
randomized data supporting an intensive management strategy, such an approach cannot be supported outside of a clinical trial. Because of the rapidly developing nature of the treatment landscape in SMM, we recommend that whenever feasible, patients should be referred to a center with a myeloma specialist and always be considered for enrollment in a clinical trial, a partial list of which is provided in supplemental eTable 1.
Submitted June 28, 2020; accepted for publication September 28, 2020

Disclosures: The authors have disclosed that they have no financial interests, arrangements, affiliations, or commercial interests with the manufacturers of any products discussed in this article or their competitors.

Funding: This work was supported by funding from the University of Wisconsin Trilium Fund.

Correspondence: Natalie S. Callander, MD, University of Wisconsin Carbone Cancer Center, 4059 WIMR, Madison, WI 53792.

Email: nsc@medicine.wisc.edu

\section{References}

1. Kaufmann SH. Immunology's foundation: the 100-year anniversary of the Nobel Prize to Paul Ehrlich and Elie Metchnikoff. Nat Immunol 2008;9: 705-712.

2. Tiselius A. Electrophoresis of serum globulin: electrophoretic analysis of normal and immune sera. Biochem J 1937;31:1464-1477.

3. Longsworth LG, Shedlovsky T, Macinnes DA. Electrophoretic patterns of normal and pathological human blood serum and plasma. J Exp Med 1939;70:399-413.

4. Waldenström J. Incipient myelomatosis or "essential" hyperglobulinemia with fibrinogenopenia-a new syndrome? Acta Med Scand 1944;117:216-247.

5. Hällén J. Discrete gammaglobulin (M-)components in serum. Clinical study of 150 subjects without myelomatosis. Acta Med Scand Suppl 1966;462:1-127.

6. Kyle RA. Monoclonal gammopathy of undetermined significance. Natural history in 241 cases. Am J Med 1978;64:814-826.

7. Rajkumar SV, Dimopoulos MA, Palumbo A, et al. International Myeloma Working Group updated criteria for the diagnosis of multiple myeloma. Lancet Oncol 2014;15:e538-548.

8. Wu SP, Minter A, Costello R, et al. MGUS prevalence in an ethnically Chinese population in Hong Kong. Blood 2013;121:2363-2364

9. Iwanaga M, Tomonaga M. Prevalence of monoclonal gammopathy of undetermined significance in Asia: a viewpoint from Nagasaki atomic bomb survivors. Clin Lymphoma Myeloma Leuk 2014;14:18-20.

10. Wadhera RK, Rajkumar SV. Prevalence of monoclonal gammopathy of undetermined significance: a systematic review. Mayo Clin Proc 2010; 85:933-942.

11. Crawford J, Eye MK, Cohen HJ. Evaluation of monoclonal gammopathies in the "well" elderly. Am J Med 1987;82:39-45.

12. Landgren $\mathrm{O}$, Graubard BI, Kumar S, et al. Prevalence of myeloma precursor state monoclonal gammopathy of undetermined significance in 12372 individuals 10-49 years old: a population-based study from the National Health and Nutrition Examination Survey. Blood Cancer J 2017; 7:e618.

13. Landgren O, Rajkumar SV, Pfeiffer RM, et al. Obesity is associated with an increased risk of monoclonal gammopathy of undetermined significance among black and white women. Blood 2010;116:1056-1059.

14. Chang SH, Luo S, Thomas TS, et al. Obesity and the transformation of monoclonal gammopathy of undetermined significance to multiple myeloma: a population-based cohort study. J Natl Cancer Inst 2016;109: djw264.

15. Grass S, Preuss KD, Ahlgrimm M, et al. Association of a dominantly inherited hyperphosphorylated paraprotein target with sporadic and familial multiple myeloma and monoclonal gammopathy of undetermined significance: a case-control study. Lancet Oncol 2009;10: 950-956.

16. Zwick C, Held G, Auth $M$, et al. Over one-third of African-American MGUS and multiple myeloma patients are carriers of hyperphosphorylated paratarg-7, an autosomal dominantly inherited risk factor for MGUS/MM. Int J Cancer 2014;135:934-938.

17. Chubb D, Weinhold N, Broderick $P$, et al. Common variation at $3 q 26.2$, $6 p 21.33,17 p 11.2$ and 22q13.1 influences multiple myeloma risk. Nat Genet 2013;45:1221-1225.

18. Landgren O, Shim YK, Michalek J, et al. Agent Orange exposure and monoclonal gammopathy of undetermined significance: an Operation Ranch Hand veteran cohort study. JAMA Oncol 2015;1:1061-1068.

19. Landgren O, Kyle RA, Hoppin JA, et al. Pesticide exposure and risk of monoclonal gammopathy of undetermined significance in the Agricultural Health Study. Blood 2009;113:6386-6391.
20. Landgren O, Zeig-Owens R, Giricz O, et al. Multiple myeloma and its precursor disease among firefighters exposed to the World Trade Center disaster. JAMA Oncol 2018;4:821-827.

21. Lynch HT, Ferrara K, Barlogie B, et al. Familial myeloma. N Engl J Med 2008;359:152-157.

22. Clay-Gilmour Al, Kumar S, Rajkumar SV, et al. Risk of MGUS in relatives of multiple myeloma cases by clinical and tumor characteristics. Leukemia 2019;33:499-507.

23. Greenberg AJ, Rajkumar SV, Vachon CM. Familial monoclonal gammopathy of undetermined significance and multiple myeloma: epidemiology, risk factors, and biological characteristics. Blood 2012;119:5359-5366.

24. VanValkenburg ME, Pruitt GI, Brill IK, et al. Family history of hematologic malignancies and risk of multiple myeloma: differences by race and clinical features. Cancer Causes Control 2016;27:81-91.

25. Weiss BM, Abadie J, Verma P, et al. A monoclonal gammopathy precedes multiple myeloma in most patients. Blood 2009;113:5418-5422.

26. Landgren O, Kyle RA, Pfeiffer RM, et al. Monoclonal gammopathy of undetermined significance (MGUS) consistently precedes multiple myeloma: a prospective study. Blood 2009;113:5412-5417.

27. Dispenzieri A, Katzmann JA, Kyle RA, et al. Prevalence and risk of progression of light-chain monoclonal gammopathy of undetermined significance: a retrospective population-based cohort study. Lancet 2010;375:1721-1728.

28. Leung N, Bridoux F, Hutchison CA, et al. Monoclonal gammopathy of renal significance: when MGUS is no longer undetermined or insignificant. Blood 2012;120:4292-4295.

29. Steiner N, Göbel G, Suchecki P, et al. Monoclonal gammopathy of renal significance (MGRS) increases the risk for progression to multiple myeloma: an observational study of 2935 MGUS patients. Oncotarget 2017;9:2344-2356

30. Amaador K, Peeters H, Minnema MC, et al. Monoclonal gammopathy of renal significance (MGRS) histopathologic classification, diagnostic workup, and therapeutic options. Neth J Med 2019;77:243-254.

31. Fermand JP, Bridoux F, Dispenzieri A, et al. Monoclonal gammopathy of clinical significance: a novel concept with therapeutic implications. Blood 2018;132:1478-1485.

32. Bustoros M, Kastritis E, Sklavenitis-Pistofidis R, et al. Bone marrow biopsy in low-risk monoclonal gammopathy of undetermined significance reveals a novel smoldering multiple myeloma risk group. Am J Hemato 2019;94:E146-149

33. Cesana C, Klersy C, Barbarano L, et al. Prognostic factors for malignant transformation in monoclonal gammopathy of undetermined significance and smoldering multiple myeloma. J Clin Oncol 2002;20:1625-1634.

34. Mailankody S, Mena E, Yuan CM, et al. Molecular and biologic markers of progression in monoclonal gammopathy of undetermined significance to multiple myeloma. Leuk Lymphoma 2010;51:2159-2170.

35. Hanamura I, Stewart JP, Huang Y, et al. Frequent gain of chromosome band 1q21 in plasma-cell dyscrasias detected by fluorescence in situ hybridization: incidence increases from MGUS to relapsed myeloma and is related to prognosis and disease progression following tandem stemcell transplantation. Blood 2006;108:1724-1732.

36. Chesi M, Bergsagel PL. Molecular pathogenesis of multiple myeloma: basic and clinical updates. Int J Hematol 2013;97:313-323.

37. Fonseca R, Bailey RJ, Ahmann GJ, et al. Genomic abnormalities in monoclonal gammopathy of undetermined significance. Blood 2002; 100:1417-1424.

38. Gleeson TG, Moriarty J, Shortt CP, et al. Accuracy of whole-body lowdose multidetector CT (WBLDCT) versus skeletal survey in the detection 
of myelomatous lesions, and correlation of disease distribution with whole-body MRI (WBMRI). Skeletal Radiol 2009;38:225-236.

39. Pawlyn C, Fowkes L, Otero S, et al. Whole-body diffusion-weighted MRI: a new gold standard for assessing disease burden in patients with multiple myeloma? Leukemia 2016;30:1446-1448

40. Ippolito D, Besostri V, Bonaffini PA, et al. Diagnostic value of whole-body low-dose computed tomography (WBLDCT) in bone lesions detection in patients with multiple myeloma (MM). Eur J Radiol 2013;82:2322-2327.

41. Jamet B, Bailly C, Carlier T, et al. Imaging of monoclonal gammopathy of undetermined significance and smoldering multiple myeloma. Cancers (Basel) 2020;12:486

42. Go RS, Rajkumar SV. How I manage monoclonal gammopathy of undetermined significance. Blood 2018;131:163-173.

43. Pepe J, Petrucci MT, Nofroni I, et al. Lumbar bone mineral density as the major factor determining increased prevalence of vertebral fractures in monoclonal gammopathy of undetermined significance. Br J Haematol 2006; 134:485-490.

44. Melton LJ III, Rajkumar SV, Khosla S, et al. Fracture risk in monoclonal gammopathy of undetermined significance. J Bone Miner Res 2004;19: 25-30.

45. Veronese $\mathrm{N}$, Luchini C, Solmi M, et al. Monoclonal gammopathy of undetermined significance and bone health outcomes: a systematic review and exploratory meta-analysis. J Bone Miner Metab 2018;36: 128-132.

46. Go RS, Gundrum JD, Neuner JM. Determining the clinical significance of monoclonal gammopathy of undetermined significance: a SEERMedicare population analysis. Clin Lymphoma Myeloma Leuk 2015;15: 177-186.e4.

47. Rajkumar SV, Kyle RA, Therneau TM, et al. Serum free light chain ratio is an independent risk factor for progression in monoclonal gammopathy of undetermined significance. Blood 2005;106:812-817.

48. Kyle RA, Benson J, Larson D, et al. IgM monoclonal gammopathy of undetermined significance and smoldering Waldenström's macroglobulinemia. Clin Lymphoma Myeloma 2009;9:17-18.

49. Go RS, Heien HC, Sangaralingham LR, et al. Estimating the risk of progression of monoclonal gammopathy of undetermined significance into lymphoplasmacytic malignancies in the United States: determining demographic differences using a national dataset [abstract]. Blood 2016; 128:Abstract 843

50. Kyle RA, Larson DR, Therneau TM, et al. Long-term follow-up of monoclonal gammopathy of undetermined significance. N Engl J Med 2018;378:241-249.

51. Dalakas MC. Advances in the diagnosis, immunopathogenesis and therapies of IgM-anti-MAG antibody-mediated neuropathies. Ther Adv Neurol Disord 2018;11:1756285617746640.

52. Kyle RA, Greipp PR. Smoldering multiple myeloma. N Engl J Med 1980; 302:1347-1349.

53. International Myeloma Working Group. Criteria for the classification of monoclonal gammopathies, multiple myeloma and related disorders: a report of the International Myeloma Working Group. Br J Haematol 2003:121:749-757.

54. Hillengass J, Fechtner K, Weber MA, et al. Prognostic significance of focal lesions in whole-body magnetic resonance imaging in patients with asymptomatic multiple myeloma. J Clin Oncol 2010;28:1606-1610.

55. Kastritis E, Moulopoulos LA, Terpos E, et al. The prognostic importance of the presence of more than one focal lesion in spine MRI of patients with asymptomatic (smoldering) multiple myeloma. Leukemia 2014;28: 2402-2403.

56. Kastritis E, Terpos E, Moulopoulos L, et al. Extensive bone marrow infiltration and abnormal free light chain ratio identifies patients with asymptomatic myeloma at high risk for progression to symptomatic disease. Leukemia 2013;27:947-953.

57. Larsen JT, Kumar SK, Dispenzieri A, et al. Serum free light chain ratio as a biomarker for high-risk smoldering multiple myeloma. Leukemia 2013; 27:941-946.

58. Rajkumar SV, Larson D, Kyle RA. Diagnosis of smoldering multiple myeloma. N Engl J Med 2011;365:474-475.

59. Kyle RA, Remstein ED, Therneau TM, et al. Clinical course and prognosis of smoldering (asymptomatic) multiple myeloma. N Engl J Med 2007; 356:2582-2590.

60. Pérez-Persona $\mathrm{E}$, Vidriales $\mathrm{MB}, \mathrm{Mateo} \mathrm{G}$, et al New criteria to identify risk of progression in monoclonal gammopathy of uncertain significance and smoldering multiple myeloma based on multiparameter flow cytometry analysis of bone marrow plasma cells. Blood 2007:110:2586-2592.
61. Dispenzieri A, Kyle RA, Katzmann JA, et al. Immunoglobulin free light chain ratio is an independent risk factor for progression of smoldering (asymptomatic) multiple myeloma. Blood 2008;111:785-789.

62. Lakshman A, Rajkumar SV, Buadi FK, et al. Risk stratification of smoldering multiple myeloma incorporating revised IMWG diagnostic criteria. Blood Cancer J 2018;8:59

63. San Miguel J, Mateos MV, Gonzalez V, et al. Updated risk stratification model for smoldering multiple myeloma (SMM) incorporating the revised IMWG diagnostic criteria [abstract]. J Clin Oncol 2019;37(Suppl): Abstract 8000

64. Neben K, Jauch A, Hielscher T, et al. Progression in smoldering myeloma is independently determined by the chromosomal abnormalities del(17p), t(4;14), gain 1q, hyperdiploidy, and tumor load. J Clin Oncol 2013;31:4325-4332.

65. Rajkumar SV, Gupta V, Fonseca R, et al. Impact of primary molecular cytogenetic abnormalities and risk of progression in smoldering multiple myeloma. Leukemia 2013;27:1738-1744.

66. Chng WJ, Huang GF, Chung TH, et al. Clinical and biological implications of MYC activation: a common difference between MGUS and newly diagnosed multiple myeloma. Leukemia 2011;25:1026-1035.

67. Shou Y, Martelli ML, Gabrea A, et al. Diverse karyotypic abnormalities of the c-myc locus associated with c-myc dysregulation and tumor progression in multiple myeloma. Proc Natl Acad Sci USA 2000;97:228-233.

68. Dhodapkar MV, Sexton R, Waheed S, et al. Clinical, genomic, and imaging predictors of myeloma progression from asymptomatic monoclonal gammopathies (SWOG S0120). Blood 2014;123:78-85.

69. Fernández de Larrea $C$, Isola I, Pereira A, et al. Evolving M-protein pattern in patients with smoldering multiple myeloma: impact on early progression. Leukemia 2018;32:1427-1434.

70. Ravi P, Kumar S, Larsen JT, et al. Evolving changes in disease biomarker and risk of early progression in smoldering multiple myeloma. Blood Cancer J 2016:6:e454.

71. Wu V, Moshier E, Leng S, et al. Risk stratification of smoldering multiple myeloma: predictive value of free light chains and group-based trajectory modeling. Blood Adv 2018;2:1470-1479.

72. Rajkumar SV, Landgren O, Mateos MV. Smoldering multiple myeloma. Blood 2015:125:3069-3075.

73. Dhodapkar MV, Geller MD, Chang DH, et al. A reversible defect in natural killer $T$ cell function characterizes the progression of premalignant to malignant multiple myeloma. J Exp Med 2003;197: 1667-1676.

74. Dhodapkar MV, Krasovsky J, Osman K, et al. Vigorous premalignancy specific effector $T$ cell response in the bone marrow of patients with monoclonal gammopathy. J Exp Med 2003;198:1753-1757.

75. Dhodapkar MV. MGUS to myeloma: a mysterious gammopathy of underexplored significance. Blood 2016;128:2599-2606.

76. Zavidij $\mathrm{O}$, Haradhvala NJ, Mouhieddine TH, et al. Single-cell RNA sequencing reveals compromised immune microenvironment in precursor stages of multiple myeloma. Nature Cancer 2020;1:493-506.

77. Bailur JK, McCachren SS, Doxie DB, et al. Early alterations in stem-like/ resident $T$ cells, innate and myeloid cells in the bone marrow in preneoplastic gammopathy. JCI Insight 2019;5:e127807.

78. Chang DH, Liu N, Klimek V, et al. Enhancement of ligand-dependent activation of human natural killer $T$ cells by lenalidomide: therapeutic implications. Blood 2006;108:618-621.

79. Galustian C, Meyer B, Labarthe MC, et al. The anti-cancer agents lenalidomide and pomalidomide inhibit the proliferation and function of T regulatory cells. Cancer Immunol Immunother 2009;58:1033-1045.

80. Lagrue K, Carisey A, Morgan DJ, et al. Lenalidomide augments actin remodeling and lowers NK-cell activation thresholds. Blood 2015;126: 50-60.

81. Krämer I, Engelhardt M, Fichtner S, et al. Lenalidomide enhances myeloma-specific T-cell responses in vivo and in vitro. Oncolmmunology 2016;5:e1139662.

82. Mateos MV, Hernández MT, Giraldo $P$, et al. Lenalidomide plus dexamethasone for high-risk smoldering multiple myeloma. N Engl J Med 2013;369:438-447

83. Lonial $S$, Jacobus $S$, Fonseca $R$, et al. Randomized trial of lenalidomide versus observation in smoldering multiple myeloma. J Clin Oncol 2020; 38:1126-1137

84. Adams HC III, Stevenaert F, Krejcik J, et al. High-parameter mass cytometry evaluation of relapsed/refractory multiple myeloma patients treated with daratumumab demonstrates immune modulation as a nove mechanism of action. Cytometry A 2018:95:279-289. 
85. de Weers M, Tai YT, van der Veer MS, et al. Daratumumab, a novel therapeutic human CD38 monoclonal antibody, induces killing of multiple myeloma and other hematological tumors. J Immunol 2011 ; 186:1840-1848

86. Krejcik J, Casneuf T, Nijhof IS, et al. Daratumumab depletes CD38+ immune regulatory cells, promotes T-cell expansion, and skews T-cell repertoire in multiple myeloma. Blood 2016;128:384-394.

87. Overdijk MB, Verploegen S, Bögels M, et al. Antibody-mediated phagocytosis contributes to the anti-tumor activity of the therapeutic antibody daratumumab in lymphoma and multiple myeloma. MAbs 2015;7:311-321.

88. Landgren CO, Chari A, Cohen YC, et al. Daratumumab monotherapy for patients with intermediate-risk or high-risk smoldering multiple myeloma: a randomized, open-label, multicenter, phase 2 study (CENTAURUS). Leukemia 2020;34:1840-1852.

89. Munshi NC, Avet-Loiseau H, Rawstron AC, et al. Association of minimal residual disease with superior survival outcomes in patients with multiple myeloma: a meta-analysis. JAMA Oncol 2017;3:28-35.

90. Perrot A, Lauwers-Cances V, Corre J, et al. Minimal residual disease negativity using deep sequencing is a major prognostic factor in multiple myeloma. Blood 2018;132:2456-2464.

91. Paiva B, Puig N, Cedena MT, et al. Measurable residual disease by nextgeneration flow cytometry in multiple myeloma. J Clin Oncol 2020;38: 784-792.

92. Korde N, Roschewski M, Zingone A, et al. Treatment with carfilzomibenalidomide-dexamethasone with lenalidomide extension in patients with smoldering or newly diagnosed multiple myeloma. JAMA Oncol 2015;1:746-754
93. Mateos MV, Martinez-Lopez J, Rodriguez Otero P, et al. Curative strategy (GEM-CESAR) for high-risk smoldering myeloma (SMM): carfilzomib, lenalidomide and dexamethasone (KRd) as induction followed by HDTASCT, consolidation with Krd and maintenance with Rd [abstract]. Blood 2019;134(Suppl 1):Abstract 781.

94. Joseph NS, Dhodapkar MV, Lonial S. The role of early intervention in high-risk smoldering myeloma. Am Soc Clin Oncol Educ Book 2020;40: 1-9.

95. Hillengass J, Usmani S, Rajkumar SV, et al. International Myeloma Working Group consensus recommendations on imaging in monoclonal plasma cell disorders. Lancet Oncol 2019;20:e302-e312.

96. Kumar SK, Callander NS, Adekola K et al. NCCN Clinical Practice Guidelines in Oncology: Multiple Myeloma. Version 3.2021. Accessed October 20, 2020. To view the most recent version, visit NCCN.org

97. Dimopoulos MA, Sonneveld P, Leung N, et al. International Myeloma Working Group recommendations for the diagnosis and management of myeloma-related renal impairment. J Clin Oncol 2016;34:1544-1557.

98. Ghobrial IM, Badros AZ, Vredenburgh JJ, et al. Phase II trial of combination of elotuzumab, lenalidomide, and dexamethasone in high-risk smoldering multiple myeloma [abstract]. Blood 2016;128:Abstract 976

99. Bustoros $M$, Nadeem $O$, Sperling AS, et al. Phase II trial of the combination of ixazomib, lenalidomide, and dexamethasone in high-risk smoldering multiple myeloma. Blood 2019;134(Suppl):Abstract 580

100. Manasanch EE, Jagannath $\mathrm{S}$, Lee HC, et al. A multicenter phase II single arm trial of isatuximab in patients with high risk smoldering multiple myeloma (HRSMM) [abstract]. Blood 2019;134(Suppl):Abstract 3116

\title{
Explore Oncology From Every Angle
}

\author{
The Hub for Disease-Specific Clinical Information \\ > JNCCN Spotlights: \\ exclusive commentary \\ about current therapies \\ Video interviews with experts \\ $>$ Links to professional \\ and patient resources \\ $>$ Summaries of news \\ and medical literature
}

\section{JNCCN360.org}

WIARBORSIDE

The nexus of knowledge
NCCN
$\begin{aligned} & \text { National } \\ & \text { Comprehensive } \\ & \text { Cancer } \\ & \text { Network }\end{aligned}$ 
Supplemental online content for:

\section{Diagnosis and Management of Monoclonal Gammopathy and Smoldering Multiple Myeloma}

Timothy M. Schmidt, MD, and Natalie S. Callander, MD

J Natl Compr Canc Netw 2020;18(12):1720-1729

eTable 1: Active Phase II/III Interventional Trials in Smoldering Multiple Myeloma 


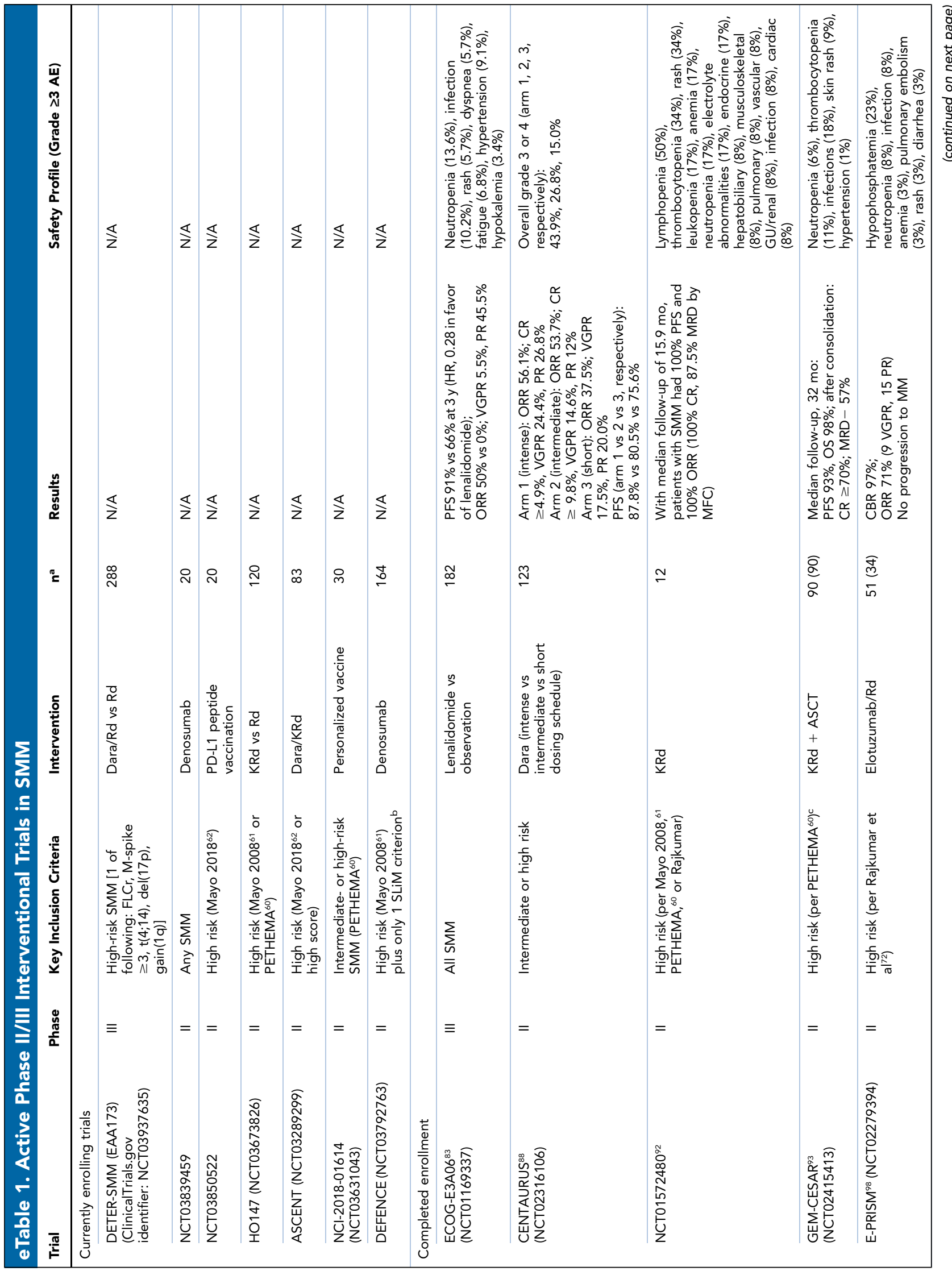




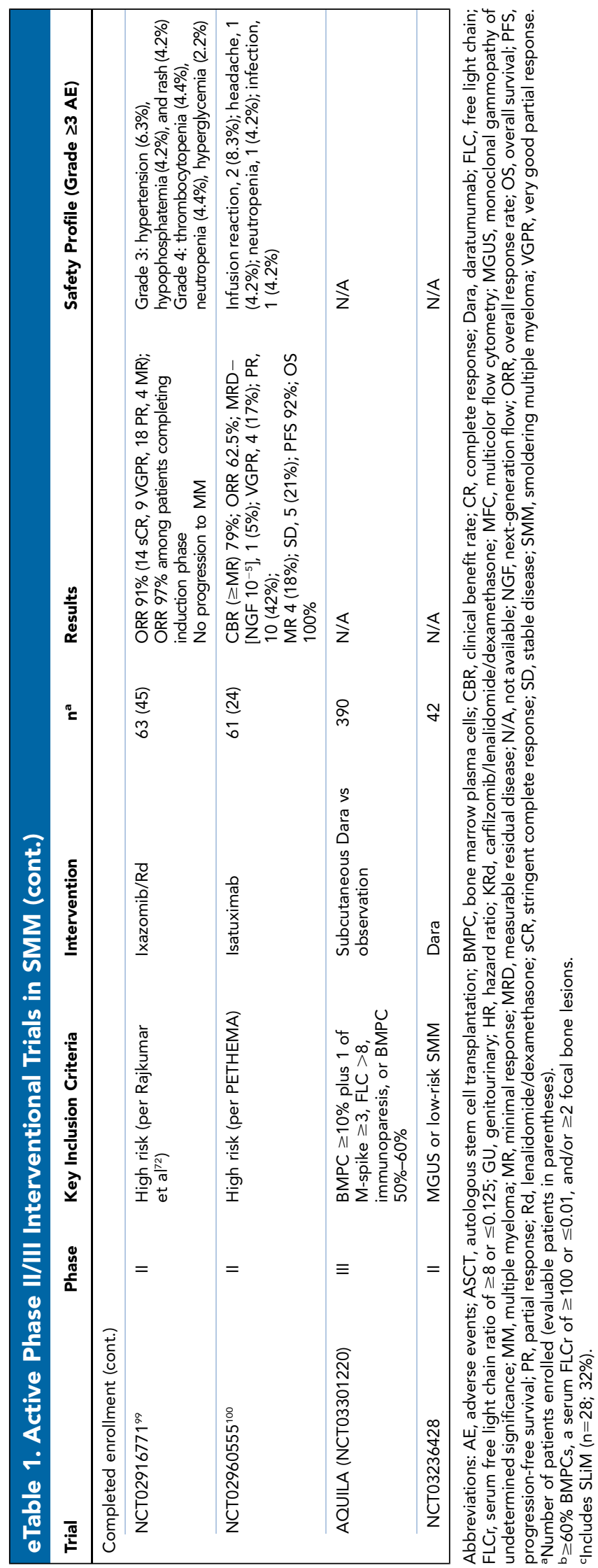

